\title{
Combination Detergent/MALDI Matrix: Functional Cleavable Detergents for Mass Spectrometry
}

\author{
Jeremy L. Norris, Ned A. Porter, Richard M. Caprioli* \\ Departments of Chemistry and Biochemistry, Mass Spectrometry Research Center, \\ Vanderbilt University, Room 9160, Medical research Building 3, $46521^{\text {st }}$ Avenue South, \\ Nashville, TN 37232-8575.
}

Synthesis. Synthesis of cleavable detergents was carried out using the synthetic methods outlined below. All reagents were purchased from Sigma-Aldrich (St. Louis, MO) and used as indicated.

Chloromethyldodecyl ether (4). Preparation of this compound was accomplished using previously described methodology. ${ }^{1}$ A volume of $5 \mathrm{~mL}(39.4 \mathrm{mmol})$ of chlorotrimethylsilane (TMS-Cl) and $0.3 \mathrm{~g}$ of paraformaldehyde were placed in a flame dried $25 \mathrm{~mL}$ round bottom flask. The reagents were allowed to stir under inert atmosphere until homogeneous. A volume of $2.27 \mathrm{~mL}$ (10 mmol) of $n$-dodecanol (3) were added drop wise to the reaction vessel. The reagents react at room temperature for a period of two hours. The TMS-Cl is removed under vacuum followed by a vacuum distillation of the product, chloromethyldodecyl ether (4). A total of $0.973 \mathrm{~g} \mathrm{(41 \%} \mathrm{yield)}$ of product were collected at $106^{\circ}-109^{\circ} \mathrm{C}$ at 0.4 torr. ${ }^{1} \mathrm{H}$ NMR $\left(300 \mathrm{MHz}, \mathrm{CDCl}_{3}\right)$ : $\delta=$ $5.53\left(\mathrm{~s}, 2 \mathrm{H} ; \mathrm{OCH}_{2} \mathrm{Cl}\right), \delta=3.65\left(\mathrm{t}, 2 \mathrm{H} ; \mathrm{CH}_{2} \mathrm{CH}_{2} \mathrm{O}\right), \delta=1.60\left(\mathrm{~m}, 2 \mathrm{H} ; \mathrm{CH}_{2} \mathrm{CH}_{2} \mathrm{CH}_{2} \mathrm{O}\right), \delta=$ $1.24(\mathrm{~m}, 18 \mathrm{H} ; \mathrm{Alk}), \delta=0.85\left(\mathrm{t}, 3 \mathrm{H} ; \mathrm{CH}_{2} \mathrm{CH}_{2} \mathrm{CH}_{3}\right) .{ }^{13} \mathrm{C} \mathrm{NMR}\left(300 \mathrm{MHz}, \mathrm{CDCl}_{3}\right): \delta=$ 83.3, 70.7, 31.9, 29.6(m), 29.3, 29.1, 28.9, 25.9, 22.7, 14.1 .

2-cyano-3-(4-dodecyloxymethoxyphenyl)-acrylic acid (1). Powdered $\mathrm{NaOH}(107 \mathrm{mg}$, $2.68 \mathrm{mmol}$ ) was dissolved in $2 \mathrm{~mL}$ of dimethylsulfoxide (DMSO) in a flame dried $25 \mathrm{~mL}$ round bottom flask. To this mixture, $0.302 \mathrm{~g}(1.35 \mathrm{mmol})$ of $\alpha$-cyano-4-hydroxycinnamic acid (5) was added to the reaction. The reaction mixture was placed under inert atmosphere and allowed to stir until all reagents were dissolved. At this time, $0.255 \mathrm{~g}$ $(1.08 \mathrm{mmol})$ of the newly synthesized chloromethyl ether (4) was added drop wise to the reaction mixture. The reaction was allowed to stir for a period of 12-16 hours. TLC confirmed that the reaction was complete. Reaction mixture was diluted with chloroform and washed repeatedly with saturated $\mathrm{NaCl}$. Remaining traces of DMSO were removed under vacuum with an in-line cold trap. A weight of $0.653 \mathrm{~g}(65.3 \%$ yield $)$ of product (1) was purified. ${ }^{1} \mathrm{H}$ NMR $\left(300 \mathrm{MHz}, \mathrm{CDCl}_{3}\right): \delta=10.86(\mathrm{~s}, 1 \mathrm{H} ; \mathrm{C}(\mathrm{O}) \mathrm{OH}), \delta=8.19(\mathrm{~s}, 1 \mathrm{H}$; $\operatorname{ArCH}=\mathrm{C}), \delta=7.97(\mathrm{~d}, J=8.7 \mathrm{~Hz}, 2 \mathrm{H} ; \mathrm{ArH}), \delta=7.12(\mathrm{~d}, J=8.7 \mathrm{~Hz}, 2 \mathrm{H} ; \mathrm{ArH}), \delta=5.47$ $\left(\mathrm{s}, 2 \mathrm{H} ; \mathrm{OCH}_{2} \mathrm{O}\right), \delta=3.68\left(\mathrm{t}, 2 \mathrm{H} ; \mathrm{OCH}_{2} \mathrm{CH}_{2}\right), \delta=1.60\left(\mathrm{~m}, 2 \mathrm{H} ; \mathrm{OCH}_{2} \mathrm{CH}_{2} \mathrm{CH}_{2}\right), \delta=1.22$ $(\mathrm{m}, 18 \mathrm{H} ; \mathrm{Alk}), \delta=0.86\left(\mathrm{t}, 3 \mathrm{H} ; \mathrm{MeCH}_{2}\right) .{ }^{13} \mathrm{C} \mathrm{NMR}\left(300 \mathrm{MHz}, \mathrm{CDCl}_{3}\right): \delta=163.1,162.3$, 155.2 , 134.2, 122.5, 116.4, 116.3, 96.7, 90.5, 79.4, 79.0, 78.5, 69.9, 31.3, 29.0, 28.7, 28.6, 25.4, 22.1, 13.9. EIMS: $387.2\left(\mathrm{M}^{+\bullet}\right), 343.2\left({\left.\mathrm{M}-\mathrm{CO}_{2}\right), 313.2,199.2,145.0 .}\right.$ HRMS (ESI) calcd. for $\mathrm{C}_{23} \mathrm{H}_{33} \mathrm{NO}_{4}(\mathrm{M}+\mathrm{Na})^{+}, 410.2302$; found, 410.2272 . 
Bromo-acetic acid 2-trimethylsilanyl-ethyl ester (8). Preparation of this compound was accomplished using previously described methodology. ${ }^{2}$ In a dry $50 \mathrm{~mL}$ round bottom flask, $1.65 \mathrm{~mL}(11.54 \mathrm{mmol})$ of trimethylsilyl ethanol (7), $0.88 \mathrm{~mL}(11 \mathrm{mmol})$ of pyridine, $0.124 \mathrm{~g}(1 \mathrm{mmol})$ of N,N-dimethylaminopyridine, and $20 \mathrm{~mL}$ of methylene chloride were placed. The reaction mixture was placed under inert atmosphere. A volume of $0.97 \mathrm{~mL}$ bromoacetyl bromide (6) was added drop wise to the reaction mixture. The reaction proceeded for two hours at room temperature. Reaction was washed twice with 1 $\mathrm{M} \mathrm{HCl}$ followed by a wash with saturated $\mathrm{NaCl}$. The organic layer was dried over $\mathrm{MgSO}_{4}$. A quantitative yield was obtained, $2.74 \mathrm{~g}$ of trimethylsilylethyl bromoacetate (8). ${ }^{1} \mathrm{H}$ NMR $\left(300 \mathrm{MHz}, \mathrm{CDCl}_{3}\right): \delta=4.22\left(\mathrm{t}, 2 \mathrm{H} ; \mathrm{OCH}_{2} \mathrm{CH}_{2}\right), \delta=3.77\left(\mathrm{~s}, 2 \mathrm{H} ; \mathrm{BrCH}_{2} \mathrm{C}(\mathrm{O})\right)$, $\delta=0.99\left(\mathrm{t}, 2 \mathrm{H} ; \mathrm{CH}_{2} \mathrm{CH}_{2} \mathrm{Si}\right), \delta=-0.02(\mathrm{~s}, 9 \mathrm{H} ; \mathrm{SiMe}) .{ }^{13} \mathrm{C} \mathrm{NMR}\left(300 \mathrm{MHz}, \mathrm{CDCl}_{3}\right): \delta=$ $167.2,64.7,26.1,17.1,-1.56$.

(2'-Trimethylsilyl)ethoxycarbonylmethylen-triphenylphosphonium bromide (9). Preparation of this compound was accomplished using previously described methodology. ${ }^{3}$ To the $2.74 \mathrm{~g}(11.46 \mathrm{mmol})$ of trimethylsilylethyl bromoacetate (8) previously synthesized, $4.51 \mathrm{~g}(17.20 \mathrm{mmol})$ of triphenylphosphine and $20 \mathrm{~mL}$ of ethyl acetate were added. The reagents were stirred at room temperature for 24 hours. A white precipitate formed which was isolated by vacuum filtration. A mass of $4.08 \mathrm{~g}(8.14$ mmol, $71.0 \%$ yield) of phosphonium salt (9) was isolated. ${ }^{1} \mathrm{H}$ NMR $\left(300 \mathrm{MHz}, \mathrm{CDCl}_{3}\right): \delta$ $=7.80(\mathrm{~m}, 15 \mathrm{H} ; \mathrm{ArH}), \delta=5.57\left(\mathrm{~d}, 2 \mathrm{H} ; \mathrm{P}^{+} \mathrm{CH}_{2} \mathrm{C}(\mathrm{O})\right), \delta=4.02\left(\mathrm{t}, 2 \mathrm{H} ; \mathrm{CH}_{2} \mathrm{CH}_{2} \mathrm{O}\right), \delta=$ $0.77\left(\mathrm{t}, 2 \mathrm{H} ; \mathrm{CH}_{2} \mathrm{CH}_{2} \mathrm{Si}\right), \delta=-0.07$ (s, 9H; SiMe $) .{ }^{13} \mathrm{C} \mathrm{NMR}\left(300 \mathrm{MHz}, \mathrm{CDCl}_{3}\right): \delta=$ 164.1, 135.0 (d, $J=11.7 \mathrm{~Hz}), 134.1$ (d, $J=43 \mathrm{~Hz}), 130.26,130.09,118.1$ (d), 65.4, 17.1, -1.7. ${ }^{31} \mathrm{P} \mathrm{NMR}\left(300 \mathrm{MHz}, \mathrm{CDCl}_{3}\right): \delta=22.2$.

3-(4-Hydroxy-3,5-dimethoxy-phenyl)-acrylic acid 2-trimethylsilanyl-ethyl ester (11). An amount of $0.824 \mathrm{~g}(1.65 \mathrm{mmol})$ of the phosphonium salt (9) previously synthesized was added to $0.23 \mathrm{~mL}(1.65 \mathrm{mmol})$ of trimethylamine in $5 \mathrm{~mL}$ of benzene. A yellow solution of ylide formed within 30 minutes. Syringaldehyde (10) (0.274 g, $1.5 \mathrm{mmol})$ was added to the ylide and stirred for 16 hours. The organic layer was washed with $1 \mathrm{M} \mathrm{HCl}$. Further purification was accomplished using flash chromatography (3:2 ethyl acetate / hexane). A yield of $73.1 \%(0.356 \mathrm{~g})$ of protected sinapinic acid (11) was obtained. ${ }^{1} \mathrm{H}$ NMR $\left(300 \mathrm{MHz}, \mathrm{CDCl}_{3}\right): \delta=7.58(\mathrm{~d}, J=16 \mathrm{~Hz}, 1 \mathrm{H} ; \mathrm{CH}=\mathrm{CHC}(\mathrm{O})), \delta=6.74(\mathrm{~s}, 2 \mathrm{H}$; $\mathrm{Ar} H), \delta=6.26\left(\mathrm{~d}, 1 \mathrm{H} ; \mathrm{CH}_{2} \mathrm{CH}_{2} \mathrm{C}(\mathrm{O})\right), \delta=5.75(\mathrm{~s}, 1 \mathrm{H} ; \mathrm{ArOH}), \delta=4.26(\mathrm{t}, 2 \mathrm{H}$; $\left.\mathrm{C}(\mathrm{O}) \mathrm{OCH}_{2} \mathrm{CH}_{2}\right), \delta=3.89(\mathrm{~s}, 6 \mathrm{H} ; \mathrm{ArOMe}), \delta=1.04\left(\mathrm{t}, 2 \mathrm{H} ; \mathrm{CH}_{2} \mathrm{CH}_{2} \mathrm{Si}\right), \delta=0.04(\mathrm{~s}, 9 \mathrm{H}$; $\left.\mathrm{Si} M e_{3}\right) .{ }^{13} \mathrm{C} \mathrm{NMR}\left(300 \mathrm{MHz}, \mathrm{CDCl}_{3}\right): \delta=167.3,147.1,144.7,137.0,125.9,116.2$, $104.9,62.6,56.3,17.4,-1.5$.

3-(4-Dodecyloxymethoxy-3,5-dimethoxy-phenyl)-acrylic acid (2). In a dry round bottom flask, $0.2 \mathrm{~mL}(1.43 \mathrm{mmol})$ of triethylamine, $0.308 \mathrm{~g}(0.951 \mathrm{mmol})$ of 3-(4-Hydroxy-3,5dimethoxy-phenyl)-acrylic acid 2-trimethylsilanyl-ethyl ester (11) and $0.271 \mathrm{~g}$ (0.836 mmol) of the newly synthesized chloromethyl ether (4) were placed. The reaction was stirred for 12 hours at room temperature. Product 30 was purified using alumina flash chromatography with methylene chloride as the mobile phase. An amount of $0.402 \mathrm{~g}$ (0.770 mmol, $92.1 \%$ yield) of compound 12 was isolated. ${ }^{1} \mathrm{H}$ NMR $\left(300 \mathrm{MHz}, \mathrm{CDCl}_{3}\right)$ : $\delta=7.58(\mathrm{~d}, J=16 \mathrm{~Hz}, 1 \mathrm{H} ; \mathrm{CH}=\mathrm{CHC}(\mathrm{O})), \delta=6.73(\mathrm{~s}, 2 \mathrm{H} ; \operatorname{Ar} H), \delta=6.30(\mathrm{~d}, 1 \mathrm{H}$; $\left.\mathrm{CH}_{2} \mathrm{CH}_{2} \mathrm{C}(\mathrm{O})\right), \delta=5.18\left(\mathrm{~s}, 1 \mathrm{H} ; \mathrm{OCH}_{2} \mathrm{O}\right), \delta=4.28\left(\mathrm{t}, 2 \mathrm{H} ; \mathrm{C}(\mathrm{O}) \mathrm{OCH}_{2} \mathrm{CH}_{2}\right), \delta=3.84(\mathrm{~s}$, 
$6 \mathrm{H} ; \mathrm{ArOMe}), \delta=3.79\left(\mathrm{t}, 2 \mathrm{H} ; \mathrm{CH}_{2} \mathrm{CH}_{2} \mathrm{O}\right), \delta=1.55\left(\mathrm{~m}, 2 \mathrm{H} ; \mathrm{CH}_{2} \mathrm{CH}_{2} \mathrm{CH}_{2} \mathrm{O}\right), \delta=1.23(\mathrm{~m}$, $18 \mathrm{H} ; \mathrm{Alk}), \delta=1.05\left(\mathrm{t}, 2 \mathrm{H} ; \mathrm{CH}_{2} \mathrm{CH}_{2} \mathrm{Si}\right), \delta=0.86\left(\mathrm{t}, 3 \mathrm{H} ; \mathrm{CH}_{2} \mathrm{Me}\right) .{ }^{13} \mathrm{C}$ NMR $(300 \mathrm{MHz}$, $\left.\mathrm{CDCl}_{3}\right): \delta=167.1,153.5,144.5,136.4,130.3,117.7,105.0,96.8,69.8,62.7,56.0,31.9$, $29.6,29.5(\mathrm{~m}), 29.3,26.1,22.7,17.4,14.1,-1.5$.

Deprotection of Compound 2. To $0.402 \mathrm{~g}(0.770 \mathrm{mmol})$ of compound 12 in $2 \mathrm{~mL}$ of tetrahydrofuran, $0.65 \mathrm{~g}$ of tetrabutylammonium fluoride was added. An immediate yellow color was observed. The reaction proceeded at room temperature for one hour. The product was extracted from saturated ammonium chloride with methylene chloride. An amount of $0.320 \mathrm{~g}(0.762 \mathrm{mmol}, 99.0 \%$ yield $)$ of Synthesis of 3-(4-Dodecyloxymethoxy3,5-dimethoxy-phenyl)-acrylic acid (2) was obtained. ${ }^{1} \mathrm{H}$ NMR $\left(300 \mathrm{MHz}, \mathrm{CDCl}_{3}\right): \delta=$ $7.66(\mathrm{~d}, J=16 \mathrm{~Hz}, 1 \mathrm{H} ; \mathrm{CH}=\mathrm{CHC}(\mathrm{O})), \delta=6.73(\mathrm{~s}, 2 \mathrm{H} ; \mathrm{ArH}), \delta=6.36(\mathrm{~d}, J=16 \mathrm{~Hz} 1 \mathrm{H}$; $\left.\mathrm{CH}_{2} \mathrm{CH}_{2} \mathrm{C}(\mathrm{O})\right), \delta=5.19\left(\mathrm{~s}, 1 \mathrm{H} ; \mathrm{OCH}_{2} \mathrm{O}\right), \delta=3.85(\mathrm{~s}, 6 \mathrm{H} ; \operatorname{ArOMe}), \delta=3.79(\mathrm{t}, 2 \mathrm{H}$; $\left.\mathrm{CH}_{2} \mathrm{CH}_{2} \mathrm{O}\right), \delta=1.55\left(\mathrm{~m}, 2 \mathrm{H} ; \mathrm{CH}_{2} \mathrm{CH}_{2} \mathrm{CH}_{2} \mathrm{O}\right), \delta=1.20$ (m, $\left.18 \mathrm{H} ; \mathrm{Alk}\right), \delta=0.86$ (t, $3 \mathrm{H}$; $\left.\mathrm{CH}_{2} \mathrm{Me}\right) .{ }^{13} \mathrm{C}$ NMR $(300 \mathrm{MHz}, \mathrm{DMSO}): \delta=167.7,153.1,143.9,135.6,130.2,118.8$, $105.5,95.8,68.6,56.0,31.27,28.8(\mathrm{~m}), 25.6,22.1,13.94,13.48$. HRMS (ESI) calcd. for $\mathrm{C}_{24} \mathrm{H}_{38} \mathrm{O}_{6}(\mathrm{M}+\mathrm{Na})^{+}, 445.2561$; found, 445.2542.

\section{References}

(1) Shipov, A. G.; Savost'yanova, I. A.; Baukov, Y. I. Zhurnal Obshchei Khimii 1989, 59, 1204-1205.

(2) Willson, T. M.; Kocienski, P.; Jarowicki, K.; Isaac, K.; Faller, A.; Campbell, S. F.; Bordner, J. Tetrahedron 1990, 46, 1757-1766.

(3) Hungerbuehler, E. v.; Seebach, D.; Wasmuth, D. Helvetica Chimica Acta 1981, $64,1467-1487$. 